Tome 43, no 24. - Mai 1936.

Mitgeteilt an der Generalversammlung der Schineizerischen zoologischen Gesellschaft in Genf, den 21. und 22. März 1936.

\title{
Stehen die Erscheinungen der Otocephalie und der Zyklopie bei Triton mit Axialgradienten oder mit Störungen bestimmter Organisatorregionen im Zusammenhang?
}

von

\section{F. E. LEHMANN}

(Zoologisches Institut der Universität Bern).

Mit 1 Textfigur.

(Ausgeführt mit Unterstützung der Stiftung Dr. Joachim de Giacomi der S.N.G.)

1. Erzeugung lokalisierter Entwicklungsstörungen im Kopfbereich durch zeitlich begrenzte Behandlung von Gastrulastadien mit LiCl.

Vor drei Jahren habe ich darüber berichtet (LEHMANN 1933, Revue Suisse de Zool. 40, S. 253), dass Zyklopie auch bei Triton durch Chlorbutol oder Phenol erzeugt werden kann. Neue Versuche mit LiCl haben insofern weiter geführt, als es mit dieser Substanz gelang, nicht nur im vorderen Kopfbereich Zyklopie, sondern ausserdem auch im hinteren Kopfbereich tiefgreifende Entwicklungsstörungen vom Typus der Otocephalie zu erzielen. Die beiden Typen, die bisher nur summarisch charakterisiert worden waren (Lehmann, Verh. d. Schweiz. Naturf. Ges. Zürich 1934), seien im Folgenden etwas ausführlicher dargestellt. Diese Befunde sollen ferner auf ihre Vereinbarkeit mit den Postulaten der Gradiententheorie geprüft werden.

A. Otocephaler Typus mit normalem Vorderende und paarigen Augen (Fig. 1). Die äussere Form des Kopfes ist normal, ebenso

Rev. SuISse de Zool., T. 43, 1936. 
die Ausdehnung der Kiemen. Im Inneren des Kopfes sind folgende Organe normal entwickelt: $a$ ) Das gesamte knorpelige Visceralcranium ist vollständig ausgebildet und zeigt im ganzen eine normale Topographie (Fig. $1 a$ u. $1 b$ ). $b$ ) Die Trabeculae cranii sind vom Opticusaustritt nach vorne normal gelagert und normal
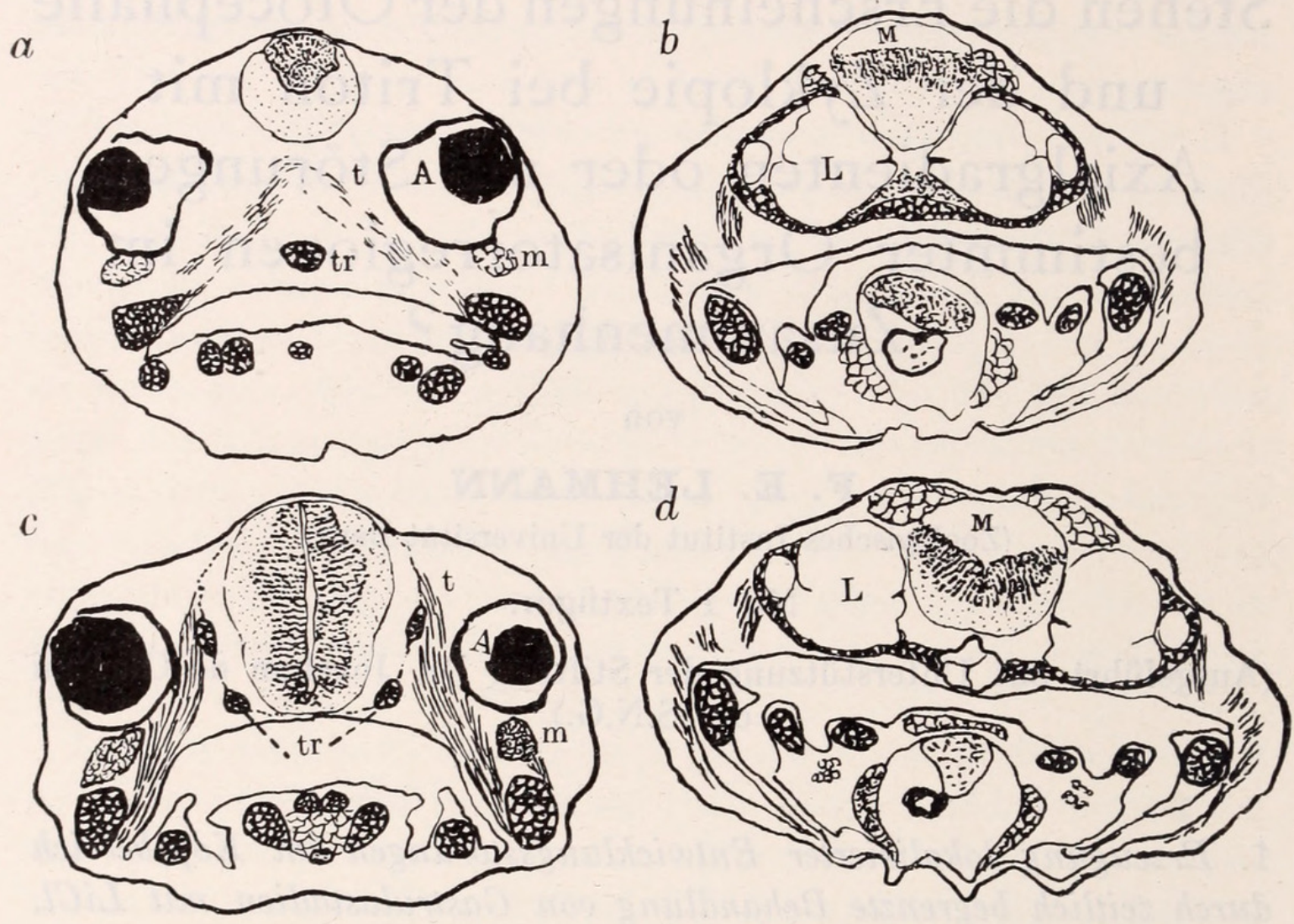

FIG. 1.

Querschnitte durch die hintere Kopfregion von Tritonlarven. $a$ und $c$ in der Region des $m$. temporalis unmittelbar hinter den Augen (A), $b$ und $d$ in der Region des Labyrinths (L). $a$ und $b$ otocephaler Typus, $c$ und $d$ normaler Vergleichskeim. Mit Tusche überzeichnete Mikrophotos. Knorpel dunkel gefeldert, Muskelquerschnitte hell gefeldert, Muskellängsschnitte gestrichelt. $\mathrm{M}=$ Medulla oblongata. Vergr. $32 \times$. Für weitere Erklärungen siehe Text.

gebaut. c) Ebenfalls von normalem Bau sind die Nasengruben, die Augen, die Bulbi olfactorii und das Vorderhirn. Sehr stark reduziert sind folgende Organe: a) Die Trabeculae cranii caudal vom Sehnervenaustritt, die hier nur einen unpaaren Stab bilden (Fig. $1 a$, tr), und die ganze Basalplatte, mitsamt dem ganzen cranialen Teil der Chorda, welche die caudalen Hirnbereiche unterlagert (Fig. 1 b). Es ist somit das ganze Knorpelskelett betroffen, 
soweit es aus der prächordalen Platte entsteht (Raven 1931, Roux'Archiv 125, S. 258). b) Stark reduziert sind die Muskeln im Kopfbereich, besonders der m. temporalis (Fig. $1 a, \mathrm{t}$ ) und m. masseter (Fig. $1 a$, m). c) Sämtliche Gehirnteile sind reduziert, soweit sie in der Region liegen, wo das Knorpelskelett defekt ist und die Chorda fehlt, also die Regionen vom Zwischenhirn (Fig. 1 a) zur Medulla oblongata (Fig. 1 $b, \mathrm{M}$ ). Der Massendefekt ist sehr stark und die Architektur der Nervensubstanz ist abnorm ("Basalmassentypus ", Lehmann 1935, Rev. Suisse de Zool. 42, S. 408). d) Die Hörblasen (Fig. $1 b, \mathrm{~L}$ ) sind in der Mediane so stark genähert, dass sie sich fast berühren und so das Bild der Otocephalie bieten.

B. Zyklopischer, bezw. synophthalmer Typus mit normaler Hörblasenregion. Die äussere Form des Kopfes ist stark verjüngt im vorderen Bereich. Auf der ventralen Seite findet sich ein unpaares Auge. Die Mundöffnung fehlt. Normalen Bau zeigen folgende Kopforgane: a) Das Chordavorderende und die parachordalen Knorpel. b) Das Gehirn von der Medulla oblongata bis zur Mittelhirnregion. c) Die Hörblasen. Abnorm ausgebildet sind folgende Organe: a) Die Knorpel des Mandibular- und Hyoidbogens sind sehr stark gestaucht und verkürzt. b) Die Trabeculae sind in ihrem vorderen Bereich durch einen unpaaren Knorpelstab repräsentiert. c) Der m. temporalis ist zum Teil median verschmolzen. d) Die Mundhöhle beginnt erst auf der Höhe des Mittelhirns. e) Die Riechgrube und der bulbus olfactorius sind unpaar und sehr klein. Das Auge ist unpaar. f) Das Vorderhirn ist stark reduziert. g) Der Hypothalamus ist schwach reduziert (vergl. dazu die Schnittbilder von zyklopischen Keimen in Adelman 1934, J. exp. Zool. 67, S. 217).

Entweder können die beiden beschriebenen Typen rein auftreten, oder kombiniert, d. h. bei den kombinierten Typen sind sämtliche Kopfbereiche abnorm. Die tabellarische Uebersicht der verschiedenen Versuchsserien zeigt, dass nach Behandlung der jüngeren Gastrulationsstadien rein otocephale oder kombinierte Typen, aber keine rein zyklopischen Typen entstehen. Nach Behandlung älterer Stadien treten rein zyklopische oder kombinierte, aber keine rein otocephalen Typen auf. Demnach liegt die kritische Phase für den hinteren Kopfbereich am Beginn der Gastrulation und ist bereits abgeschlossen, wenn das Material des vorderen Kopfbereiches maximal empfindlich ist. 


\section{TABELLE 1.}

Das Auftreten des otocephalen und des zyklopischen Typus in den verschiedenen Versuchsserien.

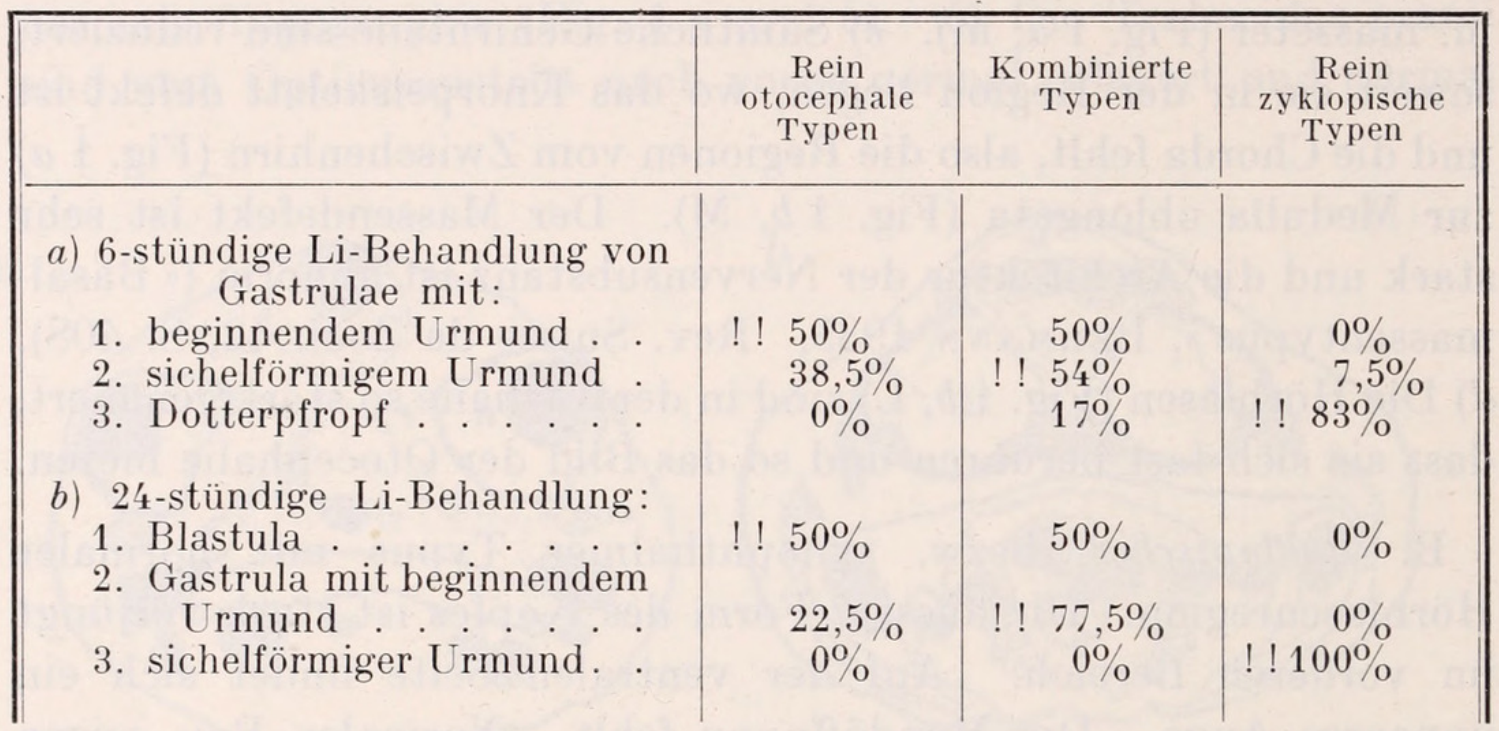

2. Zurückführung der Entwicklungsstörungen des Kopfes auf primäre Störungen des Urdarmdaches in der Kopfregion.

Die Gesamtheit der Entwicklungsstörungen des Kopfes zeigt dieselben Bilder, wie sie nach operativen Eingriffen im Bereich des vorderen Urdarmdaches erhalten werden können (LEHMAN 1926, Roux'Archiv 108, O. Mangold 1931, Erg. Biol. 7, A. Alderman 1935, J. exp. Zool. 70). Die Ausdehnung der Defekte im Nervensystem geht genau parallel mit der Ausdehnung der Störungen im Bereich der Derivate des Urdarmdaches. Die kritische Phase der Li-Empfindlichkeit liegt am Anfang der Gastrulation, also in einer Periode, wo das Ektoderm noch kaum eigene Differenzierungstendenzen besitzt, der Organisator aber bereits deutliche Anzeichen der Aufteilung in Chorda- und Somitenbereich erkennen lässt (B. Mayer 1935, Roux'Archiv 133). Diese exakte z e itliche Lokalisierung der empfindlichen Phase macht es noch wahrscheinlicher, dass die Li-Behandlung die Leistung des Kopforganisators herabsetzt und dass diese Veränderung des Organisators das Abnormwerden des Gehirns veranlasst (LEHMAN N 1936. Naturwiss., im Druck). Untersuchungen von Röнlich (Roux' Archiv 129, 1933) zeigen, dass auch in der Normalentwicklung das Urdarmdach, das den Hirnbereich unterlagert, in zwei deutlich unterscheidbare Regionen unterteilt werden kann: eine hintere 
Region, wo das mediane Material schon auf dem Neurulastadium deutlich vom unterlagernden Entoderm geschieden ist, und eine vordere Region, wo Kopfdarmdach und Mesoderm während dieser Phase in der Mediane noch eine einheitliche Platte bilden. Die Grenze zwischen beiden Bereichen liegt in der Zwischenhirnregion. Auch bei der Bildung des Kopfskeletts zeigt sich ein Unterschied zwischen den beiden Regionen (Raven 1931, l.c.). In der hinteren Region entstehen Basalplatte und Trabekel aus der prächordalen Platte, in der vorderen Region entsteht der Knorpel der Trabekel aus dem Mesektoderm. Dabei müssen wir auf Grund der Beobachtungen von Holtfreter (Roux'Archiv 129, 1933) vermuten, dass die Lage der Trabekel, wie überhaupt die Lage der aus dem Mesektoderm entstehenden Visceralknorpel durch die Architektur der entodermalen Vorderdarmwand, die nachweislich autonome Gestaltungstendenzen hat, bestimmt wird. Eine Störung in der Architektur der entodermalen Schlundbogen kann nachgewiesenermassen Grösse und Form der Knorpel mesektodermaler Herkunft beeinflussen.

Die Scheidung des Urdarmdaches im Kopfb e r e i c h lässt sich somit auf Grund morphologischer und physiologischer Kriterien durchführen; es liegen hier offenbar zwei verschiedene organbildende Territorien vor:

A. E in vorderer B e reich der 1. enger mit dem Entoderm des Vorderdarmes zusammenhängt, 2. das Vorder- und Zwischenhirn unterlagert, und 3. dessen sensible Phase gegen die Mitte der Gastrulation liegt, zugleich mit der kritischen Phase des entodermalen Schlundbogenmaterials.

B. Ein hinterer B e reich, der 1. stärker mit der Chordamesodermplatte zusammenhängt, 2. das Gehirn vom Mittelhirn bis zur Medulla oblongata unterlagert, und 3. dessen kritische Phase zu Beginn der Gastrulation liegt, gleichzeitig mit der kritischen Phase des Chordamesoderms in der vorderen Rumpfregion.

\section{Die Li-Empfindlichkeit des Organisators und die Axialgradienten.}

Ausgehend von Versuchen von Bellamy (Biol. Bull. 41, 1921. Am. J. Anat. 30, 1922), haben Child, Cotronei, Huxley und De Beer (The elements of exp. Embryol. Cambridge 1934) die 
Meinung vertreten, dass es möglich sei, sämtliche Entwicklungsstörungen, die bei Amphibienkeimen mit chemischen Mitteln erzeugt wurden, als Ausdruck eines a $\mathrm{x}$ i a le $\mathrm{n} \mathrm{Empfind-}$ lichkeitsgefälles anzusehen. Würde man im Sinne dieser Theorie annehmen, dass der Organisator im Verlaufe der Gastrulation zur sekundär-dominanten Region würde, dann wäre zu erwarten, dass zuerst die vorderste Region des Organisators die höchste Empfindlichkeit gegenüber LiCl zeigt (Vergl. HuxLEY und De Beer l.c., S. 348). Das ist nicht der Fall. Zuerst zeigt der Organisator der hinteren Kopfregion gleichzeitig mit dem vorderen Rumpforganisator eine empfindliche Periode, und dann folgt erst der vorderste Organisatorbereich. D i e s e r B e f u n d lässt sich m. E. nicht mit den Postulaten der Gradiententheorie in Einklang bringen.

Es stellt sich die Frage, aus welchen experimentellen Befunden die Postulate der Gradiententheorie für den Fall der Amphibienembryonen abgeleitet wurden. Hier scheinen sehr erhebliche Mängel in der experimentellen Fundierung der Theorie vorzuliegen. In den erwähnten Arbeiten Bellamys finden sich 1. keinerlei Angaben über die Variabilität der Reaktionen gegenüber bestimmten chemischen Einwirkungen und 2. keinerlei Angaben über den histologischen Bau der Froschembryonen, welche Missbildungen aufwiesen. Die Abbildungen und Beschreibungen von Bellamy sind so summarisch gehalten, dass aus ihnen nicht mit Sicherheit entnommen werden kann, ob in den angeführten Fällen tatsächlich ein eindeutiger Empfindlichkeitsgradient vorliegt. Dieser Mangel in der Fundierung der Gradiententheorie liegt nicht nur bei den Amphibien vor, sondern ist auch von Bäschlin (Zool. Jb. Physiol., 52 ; 1932) für die Untersuchungen an den Hydroiden aufgezeigt worden. So lange sich also die Gradiententhe ori e a $\mathrm{f}$ Material stützt, dessen morphologische und vor allem histo Jogische Charakterisierung unzureichend ist, wird sich nicht mit Sicherheit entscheiden lassen, ob die Gradiententheorie die allgemeine Geltung hat, die ihr von manchen Forschern zugeschrieben wird.

Die vorliegenden Resultate führten zu der Auffassung, dass die Erscheinungen der Zyklopie und der Otocephalie mit Störungen bestimmter Bereiche 
des Urdarmdaches in der Kopfregion zus a mmenhängen. Diese Bereiche können während kurzdauernder kritis cher Phasen durch Li-Behandlung selektiv verändert werden. Unsere Feststellungen lassen es ferner als höchst zweifelhaft erscheinen, ob sich die Gradiententheorie als Erklärungsprinzip für die geschilderten Befunde eignet. Denn einerseits ist die experimentelle Fundierung dieser Theorie durch exakte histologische Befunde noch nicht erfolgt und anderseits nimmt die ganze Konzeption der Gradiententheorie zu wenig Rücksicht auf das Vorhandensein verschiedener Embryonalbezirke mit charakteristischen Entwicklungspotenzen, so wie wir sie für die Bereiche des Kopforganisators aufzeigen konnten. 


\section{$2 \mathrm{BHL}$ Biodiversity Heritage Library}

Lehmann, F E. 1936. "Stehen die Erscheinungen der Otocephalie und der Zyklopie bei Triton mit Axialgradienten oder mit Störung bestimmter

Organisatorregionen im Zusammenhang." Revue suisse de zoologie 43, 535-541. https://doi.org/10.5962/bhl.part.117670.

View This Item Online: https://www.biodiversitylibrary.org/item/148563

DOI: https://doi.org/10.5962/bhl.part.117670

Permalink: https://www.biodiversitylibrary.org/partpdf/117670

\section{Holding Institution}

American Museum of Natural History Library

\section{Sponsored by}

BHL-SIL-FEDLINK

\section{Copyright \& Reuse}

Copyright Status: In copyright. Digitized with the permission of the rights holder.

Rights Holder: Muséum d'histoire naturelle - Ville de Genève

This document was created from content at the Biodiversity Heritage Library, the world's largest open access digital library for biodiversity literature and archives. Visit BHL at https://www.biodiversitylibrary.org. 